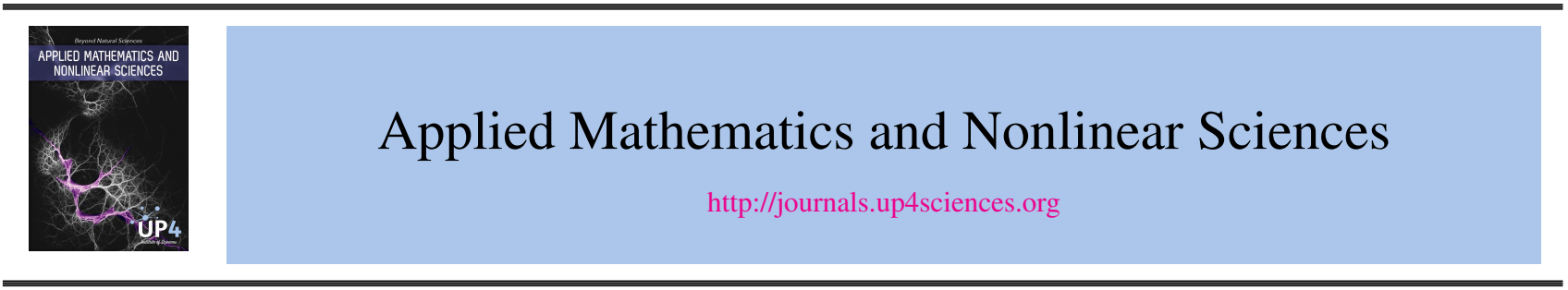

\title{
Centers: their integrability and relations with the divergence
}

\section{J. Llibre ${ }^{\dagger}$.}

Departament de Matemàtiques, Universitat Autònoma de Barcelona, 08193 Bellaterra, Barcelona, Catalonia, SPAIN

\begin{abstract}
This is a brief survey on the centers of the analytic differential systems in $\mathbb{R}^{2}$. First we consider the kind of integrability of the different types of centers, and after we analyze the focus-center problem, i.e. how to distinguish a center from a focus. This is a difficult problem which is not completely solved. We shall present some recent results using the divergence of the differential system.
\end{abstract}

Keywords and phrases: Center problem; integrability; Poincaré-Liapunov constants; divergence 2010 Mathematics Subject Classification: 34C25, 37G10, 34A34, 34C05

\section{Introduction}

This brief survey is mainly dedicated to the mathematicians which are interested in the centers of the analytic differential systems in the plane $\mathbb{R}^{2}$, and which are not specialists in this topic.

\section{Types of centers}

Let $O$ be the origin of coordinates of $\mathbb{R}^{2}$ and let $U$ be a neighborhood of $O$. We consider two real analytic functions $X(x, y)$ and $Y(x, y)$ in $U$ which vanish at $O$. In this work we deal with the analytic differential systems of the form

$$
\dot{x}=X(x, y), \quad \dot{y}=Y(x, y),
$$

where the dot denotes derivative with respect to an independent real variable $t$, usually called the time.

\section{Corresponding author.}

Email address: *jllibre@mat.uab.cat* 
When all the orbits of system (1) in a punctured neighborhood of the singular point $O$ are periodic, we say that the origin is a center. If the orbits of system (1) in a punctured neighborhood of $O$ spiral to $O$ when $t \rightarrow+\infty$ or when $t \rightarrow-\infty$, then we say that the origin is a focus. In the first case $(t \rightarrow+\infty)$, we say that it is stable and in the second case $(t \rightarrow-\infty)$, we say that it is unstable. If the origin is either a focus or a center, we say that it is a monodromic singular point. The center-focus problem consists in distinguishing when a monodromic singular point is either a center or a focus. The center-focus problem started with Poincaré [22] and Dulac [6], and in the present days many questions remains open.

If a real analytic system (1) has a center at the origin, then after a linear change of variables and a rescaling of the time variable, it can be written in one of the following three forms:

$$
\begin{aligned}
& \dot{x}=-y+X_{2}(x, y), \\
& \dot{y}=x+Y_{2}(x, y),
\end{aligned}
$$

called a linear type center,

$$
\begin{aligned}
& \dot{x}=y+X_{2}(x, y), \\
& \dot{y}=Y_{2}(x, y),
\end{aligned}
$$

called a nilpotent center,

$$
\begin{aligned}
& \dot{x}=X_{2}(x, y), \\
& \dot{y}=Y_{2}(x, y),
\end{aligned}
$$

called a degenerate center, where $X_{2}(x, y)$ and $Y_{2}(x, y)$ are real analytic functions without constant and linear terms, defined in a neighborhood of the origin.

\section{About the integrability of the centers}

The characterization of linear type centers using their first integrals is due to Poincare [22] in the case of polynomial differential systems and to Liapunov [16] in the case of analytic differential systems, see also Moussu [20].

Linear Type Center Theorem. System (2) has a center at the origin if and only if there exists a local analytic first integral of the form $H=x^{2}+y^{2}+F(x, y)$ defined in a neighborhood of the origin, where $F$ starts with terms of order higher than 2.

Using this theorem Poincaré and Liapunov [16,22] constructed an algorithm which, when it can be applied because it usually needs huge computations, solves in principle the center problem for linear type centers.

We say that a non-zero formal power series $H=\sum_{i, j=0}^{\infty} a_{i j} x^{i} y^{j}$ is a formal first integral of system (3), if it formally satisfies that

$$
\frac{\partial H}{\partial x}\left(y+X_{2}(x, y)\right)+\frac{\partial H}{\partial y} Y_{2}(x, y) \equiv 0 .
$$

The integrability of the nilpotent centers was studied in [4].

Nilpotent Center Theorem. Assume that the real analytic differential system (3) has a nilpotent center at the origin.

(a) If the system has a formal first integral, then it has a formal first integral of the form $H=y^{2}+F(x, y)$, where F starts with terms of order higher than two.

(b) If the system has a local analytic first integral defined at the origin, then it has a local analytic first integral of the form $H=y^{2}+F(x, y)$, where $F$ starts with terms of order higher than two. 
(c) If $X=y f\left(x, y^{2}\right)$ and $Y=g\left(x, y^{2}\right)$, then the system has a local analytic first integral of the form $H=$ $y^{2}+F(x, y)$, where $F$ starts with terms of order higher than two.

We note that statement (b) provides a tool for detecting if a given analytic nilpotent center has or not a local analytic first integral.

Analytic systems having a nilpotent singular point at the origin were studied by Andreev [1] in order to obtain their local phase portraits. But Andreev's results do not distinguish between a focus and a center. Takens [24] provided a normal form for nilpotent centers or foci. Moussu [19] found the $C^{\infty}$ normal form for analytic nilpotent centers. Berthier and Moussu in [3] study the reversibility of the nilpotent centers. Teixeira and Yang [25] analyse the relationship between reversibility and the center-focus problem for systems (2) and (3), written in a convenient normal form. Strózyna and Zoladek [23] gave also a good normal form for nilpotent centers.

The next proposition provides, probably, the easiest example of an analytic nilpotent center without a local analytic first integral, for a proof see Proposition 7 of [4].

Proposition 1. The vector field $\left(y+x^{2},-x^{3}\right)$ has a nilpotent center at the origin, but there is neither a local analytic first integral defined at the origin, nor a formal one.

We note that systems satisfying the assumptions of statement (c) of the Nilpotent Center Theorem are timereversible because they are invariant under the change of variables $(u, v, t) \rightarrow(u,-v,-t)$. So, from the Nilpotent Center Theorem and Proposition 1, it follows that does not exist an analytic change of variables which transforms the analytic nilpotent center of Proposition 1 into another analytic nilpotent center of the form (3) which is timereversible under the change of variables $(u, v, t) \rightarrow(u,-v,-t)$.

For degenerate analytic centers it is known that in general they have no local analytic first integrals defined in its neighborhood. Nemitskii and Stepanov in [21] (page 122) give a real polynomial differential system which has a degenerate center, but the system has neither a local analytic first integral in its neighborhood, nor a formal one. In [20] Moussu gives another example of a real polynomial differential system having a degenerate center for which does not exist a local analytic first integral. There are partial results about the center-focus problem for degenerate centers, see for instance [10].

The following result about the $C^{\infty}$ first integrals of all centers is due to Mazzi and Sabatini [18].

Theorem 2. Any analytic differential system having a center has a local $C^{\infty}$ first integral defined in its neighborhood.

About the analytic integrals of all centers there is the next result prove in [15].

Theorem 3. Any analytic differential system having a center at the point $p$ has a local analytic first integral defined in a punctured neighborhood of $p$, but such an analytic first integral does not need to be defined at the center point when the center is nilpotent or degenerate.

\section{Characteristic directions}

Assume that we have the analytic differential system

$$
\begin{aligned}
& \dot{x}=X_{n}(x, y)+O_{n+1}(x, y), \\
& \dot{y}=Y_{m}(x, y)+O_{m+1}(x, y),
\end{aligned}
$$

where $n \geq 1$ and $m \geq 1$ are integers and $X_{n}(x, y)$ and $Y_{m}(x, y)$ are non-zero homogeneous polynomials of degrees $n$ and $m$ respectively, formed by the lowest order terms of $X(x, y)$ and $Y(x, y)$ of system (1), respectively. 
Define the homogeneous polynomial

$$
\Delta(x, y)= \begin{cases}y X_{n}(x, y)-x Y_{m}(x, y) & \text { if } n=m, \\ y X_{n}(x, y) & \text { if } n<m, \\ -x Y_{m}(x, y) & \text { if } n>m .\end{cases}
$$

The real linear factors of the polynomial $\Delta(x, y)$ provide the possible directions that the trajectories can reach or exit the singular point located at the origin of coordinates of system (5). Such directions are called characteristic directions.

A sufficient condition in order that system (5) has a monodromic singular point at the origin is that $\Delta(x, y)=0$ only if $(x, y)=(0,0)$. In this case the origin has no characteristic directions.

A necessary condition in order that system (5) has a monodromic singular point at the origin is that $\Delta(x, y) \geq$ 0 or $\Delta(x, y) \leq 0$ for all $(x, y) \in \mathbb{R}^{2}$. For more details on characteristic directions see for instance [2].

\section{Poincaré-Liapunov constants}

Suppose that the analytic differential system (1) has a monodromic singular point at the origin $O$. Let $\Sigma$ be an analytic transversal section at $O$, that is, an analytic arc transverse to the flow of the system such that $O \in \partial \Sigma$, the boundary of $\Sigma$. We consider a parameter $\rho$ of $\Sigma$ such that $\rho=0$ corresponds to the origin of coordinates and $\Sigma$ is parameterized by the interval $\left(0, \rho^{*}\right)$ with $\rho^{*}>0$. Given a point $\rho$ in $\Sigma$ we consider the orbit of system (1) with $\rho$ as initial condition. Due to the fact that the origin is monodromic, if $\rho$ is close enough to $O$ and we follow the orbit for positive values of the time $t$, it will cut $\Sigma$ again at some point. We define the Poincaré map $\mathscr{P}: \Sigma \rightarrow \Sigma$ being $\mathscr{P}(\rho)$ the point in $\Sigma$ corresponding to the first cut with $\Sigma$ of the orbit through $\rho$ in positive time.

It is clear that the origin of the analytic differential system (1) is a center if and only if the Poincare map is the identity. For systems with the linear type form (2) and for systems with the nilpotent form (3), it is possible to find a parametrization of $\Sigma$ such that the Poincaré map is analytic in $\rho=0$ and writes as

$$
\mathscr{P}(\rho)=\rho+\sum_{i=3}^{\infty} \alpha_{i} \rho^{i}
$$

where $\alpha_{i}$ are algebraic expressions in the coefficients of $X_{2}$ and $Y_{2}$. There are systems with the degenerate form (4) for which such a parametrization is also possible, for instance the ones which do not have characteristic directions, see for instance [9].

The stability of the origin is clearly given by the sign of the first non-zero $\alpha_{i}$ (that is, it is unstable if $\alpha_{i}>0$, and stable if $\alpha_{i}<0$ ), and if all the $\alpha_{i}$ are zero then the origin is a center. Moreover, the even-indexed terms $\alpha_{2 k}$ are algebraic expressions of the previous $\alpha_{i}$. Therefore the interesting expressions are the ones with odd index, i.e. the $\alpha_{2 k+1}$ 's. Moreover we define the $(k+1)$-th Poincaré-Liapunov constant as the expression $\alpha_{2 k+1}$ modulus the vanishing of all the previous ones. In general the computation of the Poincaré-Liapunov constants is a very difficult computational problem. See for more details [5].

The technique of the Poincaré-Liapunov constants for solving the focus-center problem for linear type centers and of the centers without characteristic directions can be extended to nilpotent centers, see [8,11, 12].

In short, now we have algorithms based in the computation of Poincaré-Liapunov constants for solving the focus-center problem for linear type centers, nilpotent centers and centers without characteristic directions. Moreover, for such centers the Poincaré-Liapunov constants are algebraic polynomials in function of the coefficients of the polynomial differential systems.

The conditions on the coefficients of a polynomial differential system which determine when such a system has a center, which is neither of linear type, nor nilpotent and with characteristic directions, can be non-algebraic as it was proved by Ilyashenko [14], see also [17]. 


\section{The divergence}

As usual we define the divergence of system (1), and we denote it by $\operatorname{div}(x, y)$, as the function

$$
\operatorname{div}(x, y)=\frac{\partial X}{\partial x}(x, y)+\frac{\partial Y}{\partial y}(x, y) .
$$

System (1) is said to be Hamiltonian if $\operatorname{div}(x, y) \equiv 0$. In such a case there exists a neighborhood of the origin $U$ and an analytic function $H: U \subseteq \mathbb{R}^{2} \rightarrow \mathbb{R}$, called the Hamiltonian, such that

$$
X(x, y)=-\frac{\partial H}{\partial y} \quad \text { and } \quad Y(x, y)=\frac{\partial H}{\partial y} .
$$

We note that the level curves of $H$ are formed by orbits of system (1). A Hamiltonian system (1) with a monodromic singular point at $O$ necessarily has a center at the origin because the function $H$ is a first integral defined at the origin.

Our aim is to illustrate that the divergence function also can help us to solve the focus-center problem in other non-Hamiltonian differential systems.

Given a real analytic function $f: U \subseteq \mathbb{R}^{2} \rightarrow \mathbb{R}$, where $U$ is a neighborhood of the origin $O=(0,0)$, we consider its Taylor expansion at $O$, i.e.

$$
f(x, y)=f_{d}(x, y)+\mathscr{O}_{d+1}(x, y),
$$

where $d \geq 0$ is an integer and $f_{d}(x, y)$ is a non-zero homogeneous polynomial of degree $d$. We say that $f$ is positive sign definite if $f_{d}(x, y)>0$, or negative sign definite $f_{d}(x, y)<0$ for all $(x, y) \in \mathbb{R}^{2} \backslash\{(0,0)\}$. It is clear that a necessary condition for $f(x, y)$ to be of sign definite is that $d$ is even.

The next result is Proposition 1 of [13].

Proposition 4. Assume that the origin of an analytic differential system (1) is a monodromic singular point. If the divergence $\operatorname{div}(x, y)$ of system (1) is of sign definite, then the origin of system (1) is a focus; either unstable if the divergence is positive sign definite, or stable if it is negative sign definite.

We remark that in the case that the origin of system (1) is a strong focus (i.e. the eigenvalues of the linear part at the origin are of the form $\alpha+i \beta$ with $\alpha \neq 0)$, then the divergence $\operatorname{div}(0,0) \neq 0$ and the stability of the focus is given by the sign of the number $\operatorname{div}(0,0)$. The previous proposition is a generalization of this fact to any monodromic singular point. See for instance Theorem 2.15 of [7], for a proof of this classical result.

There are several tools for determining whether the origin of system (1) is monodromic. In the case that the linear part of system (1) has two complex conjugate eigenvalues with non-zero imaginary part, we have that the origin is monodromic and it can be written in the linear type form (2). Any other configuration of non-zero eigenvalues implies that the origin is not monodromic, see again for more details Theorem 2.15 of [7].

If the linear part of system (1) is not identically zero and has two zero eigenvalues, then we can decide when the origin is a monodromic singular point by the Andreev's theorem (see [1], or Theorem 3.5 of [7]), and the system can be written in the nilpotent form (3).

Finally, when the linear part of system (1) is identically zero, then the system writes in the degenerate form (4). Using the blow-up technique one can determine whether the origin is monodromic, see for instance Chapter 3 of [7].

\section{Linear type centers and their divergence}

The next result is Theorem 2 of [13]. 
Theorem 5. Consider an analytic differential system (1) whose origin is of linear type. Denote by $\operatorname{div}_{d}(x, y)$ the lowest order terms of the divergence $\operatorname{div}(x, y)$ of the system. Assume that

$$
\alpha_{d+1}=\frac{1}{d+2} \int_{0}^{2 \pi} \operatorname{div}_{d}(\cos t, \sin t) d t \neq 0 .
$$

Then the origin is a focus whose first non-zero Poincaré-Liapunov constant is $\alpha_{d+1}$.

We remark that Theorem 5 can only give a nonzero $\alpha_{d+1}$ when the $d$ in Theorem 5 is even.

The following result follows easily from Theorem 5 .

Corollary 6. Consider the system

$$
\dot{x}=-y+P_{s}(x, y), \quad \dot{y}=x+Q_{s}(x, y),
$$

where $P_{s}(x, y)$ and $Q_{s}(x, y)$ are homogeneous polynomials of odd degree s. The first Poincaré-Liapunov constants of system (7) are $\alpha_{i}=0$ for $i=1,2, \ldots, s-1$ and

$$
\alpha_{s}=\frac{1}{s+1} \int_{0}^{2 \pi} \operatorname{div}(\cos t, \sin t) d t
$$

\section{Nilpotent centers and their divergence}

The following result is Theorem 4 of [13].

Theorem 7. Consider an analytic differential system (3) whose origin is a nilpotent singular point. Denote by $\operatorname{div}_{d}(x, y)$ the lowest order terms of the divergence $\operatorname{div}(x, y)$ of the system. Define

$$
V_{d+1}(\varepsilon)=\int_{0}^{2 \pi / \sqrt{\varepsilon}} \operatorname{div}_{d}(\cos (\sqrt{\varepsilon} t),-\sqrt{\varepsilon} \sin (\sqrt{\varepsilon} t)) d t
$$

where $\varepsilon>0$ and define the constant $v_{d+1}$ by the expansion in power series of $\sqrt{\varepsilon}$ of the function $V_{d+1}(\varepsilon)=$ $\frac{v_{d+1}}{\sqrt{\varepsilon}}+O(1)$.

(a) If the origin is a center, then $v_{d+1}=0$.

(b) If $v_{d+1}>0$ (resp. $\left.v_{d+1}<0\right)$, then the origin is an unstable (resp. stable) focus.

\section{Centers and their divergence}

Our next main result deals with a system of linear type (2) or in degenerate form (4) with a monodromic singular point at the origin.

We assume that the origin has no characteristic directions. As we have already stated, this means that the polynomial $\Delta(x, y)$ defined in (6) is such that $\Delta(x, y)=0$ only if $(x, y)=(0,0)$. We remark that in this case the degree of the lowest order terms of $\dot{x}$ and $\dot{y}$ must coincide, that is, $X(x, y)=X_{n}(x, y)+O_{n+1}(x, y)$ and $Y(x, y)=Y_{n}(x, y)+O_{n+1}(x, y)$ where $X_{n}(x, y)$ and $Y_{n}(x, y)$ are nonzero homogeneous polynomials of degree $n$ formed by all the terms of this degree in $X(x, y)$ and $Y(x, y)$. We define

$$
v(\theta)=\exp \left[\int_{0}^{\theta} \frac{\cos \sigma P_{n}(\cos \sigma, \sin \sigma)+\sin \sigma Q_{n}(\cos \sigma, \sin \sigma)}{\cos \sigma Q_{n}(\cos \sigma, \sin \sigma)-\sin \sigma P_{n}(\cos \sigma, \sin \sigma)} d \sigma\right] .
$$

The following result is Theorem 11 of [13]. 
Theorem 8. Consider an analytic differential system (1) whose origin is monodromic and has no characteristic directions. Denote by $\operatorname{div}_{d}(x, y)$ the lowest order terms of degree $d$ of the divergence $\operatorname{div}(x, y)$ of the system. Assume that $v(2 \pi)=1$ and

$$
\alpha=\int_{0}^{2 \pi} \frac{\operatorname{div}_{d}(\cos \theta, \sin \theta) v(\theta)^{d-n+1}}{\cos \theta Q_{n}(\cos \theta, \sin \theta)-\sin \theta P_{n}(\cos \theta, \sin \theta)} d \theta \neq 0 .
$$

Then the origin is a focus which is stable (resp. unstable) if $\alpha<0$ (resp. $\alpha>0)$.

We remark that Theorem 5 is a particular case of Theorem 8. In Theorem 5 we compute the exact value of a Poincaré-Liapunov constant for a system of linear type whereas Theorem 8 also deals with systems in degenerate form.

We also remark that, as it was shown in the proof of Theorem 8 , if $v(2 \pi)>1$ then the origin is an unstable focus and if $v(2 \pi)<1$ then the origin is a stable focus. The statement of Theorem 8 can be useful to establish the stability of the origin in case that $v(2 \pi)=1$.

The following example is a particular case of Example 3 in [9], the stability of the origin can be studied using Theorem 8, for more details see [13].

Example 9. Consider the degenerate singular point at the origin of the system

$$
\begin{aligned}
& \dot{x}=-y\left(x^{2}+y^{2}\right)+x^{3}\left(\lambda_{1} x^{2}+\lambda_{2}\left(x^{2}+y^{2}\right)\right), \\
& \dot{y}=x\left(x^{2}+y^{2}\right)+x^{2} y\left(\lambda_{1} x^{2}+\lambda_{2}\left(x^{2}+y^{2}\right)\right),
\end{aligned}
$$

where $\lambda_{1}, \lambda_{2} \in \mathbb{R}$. If $3 \lambda_{1}+4 \lambda_{2}>0$ (resp. $3 \lambda_{1}+4 \lambda_{2}<0$ ), then the origin of system (8) is an unstable (resp. stable) focus.

\section{Acknowledgements}

The author is partially supported by a MINECO grant MTM2013-40998-P, an AGAUR grant number 2014SGR568, and the grants FP7-PEOPLE-2012-IRSES 318999 and 316338.

\section{References}

[1] A. Andreev, (1958), Investigation of the behaviour of the integral curves of a system of two differential equations in the neighborhood of a singular point, Translation of Amer. Math. Soc., 8, 187-207.

[2] A.A. Andronov, E.A. Leontovich, I.I. Gordon and A.G. Maier, (1973), Theory of Bifurcations of Dynamic Systems on a Plane, John Wiley and Sons, New York.

[3] M. Berthier and R. Moussu, (1994), Réversibilité et classification des centres nilpotents, Ann. Inst. Fourier (Grenoble), 44, 465-494. doi 10.5802/aif.1406

[4] J. Chavarriga, H. Giacomini, J. Giné and J. Llibre, (2003), Local analytic integrability for nilpotent centers, Ergodic Theory and Dynamical Systems, 23, 417-428. doi 10.1017/S014338570200127X

[5] A. Cima, A. Gasull, V. Mañosa and F. Mañosas, (1997), Algebraic properties of the Liapunov and period constants, Rocky Mountain J. Math., 27, 471-501. doi 10.1216/rmjm/1181071923

[6] H. Dulac, (1908), Détermination et integration d'une certaine classe d'équations différentielle ayant par point singulier un centre, Bull. Sci. Math. Sér. (2), 32, 230-252.

[7] F. Dumortier, J. Llibre and J.C. Artés, (2006), Qualitative theory of planar differential systems. Universitext. Springer Verlag, Berlin. doi 10.1007/978-3-540-32902-2

[8] I.A. García, H. Giacomini, J. Giné and J. Llibre, Analytic nilpotent centers as limits of nondegenerated centers revisited. Preprint.

[9] I.A. García, H. Giacomini and M. Grau, (2011), Generalized Hopf bifurcation for planar vector fields via the inverse integrating factor, J. Dynam. Differential Equations, 23, 251-281. doi 10.1007/s10884-011-9209-2 
[10] A. Gasull, J. Llibre, V. Man̄osa and F. Man̄osas, (2000), The focus-center problem for a type of degenerate systems, Nonlinearity, 13, 699-730. doi 10.1088/0951-7715/13/3/311

[11] H. Giacomini, J. Giné and J. Llibre, (2006), The problem of distinguishing between a center and a focus for nilpotent and degenerate analytic systems, J. Differential Equations, 227, 406-426. doi 10.1016/j.jde.2006.03.012

[12] J. Giné and J. Llibre, (2014), A method for characterizing nilpotent centers. J. Math. Anal. Appl., 413, 537-545. doi 10.1016/j.jmaa.2013.12.013

[13] M. Grau and J. Llibre, (2015), Divergence and Poincaré-Liapunov constants for analytic differential systems, J. Differential Equations, 258, 4348-4367. doi 10.1016/j.jde.2015.01.035

[14] Yu. S. Il'yashenko, (1972), Algebraic unsolvability and almost algebraic solvability of the problem for the center-focus, Funkcion. Anal. Priloz., 6, No 3, 30-37.

[15] Weigu Li, J. Llibre, M. Nicolau and Xiang Zhang, (2002), On the differentiability of first integrals of two dimensional flows, Proc. Amer. Math. Soc., 130, 2079-2088. doi 10.1090/S0002-9939-02-06310-4

[16] M.A. Liapunov, (1947) Problème général de la stabilité du mouvement, Ann. of Math. Stud. 17, Princeton University Press.

[17] J. Llibre and H. Zoladek, (2008), The Poincaré center problem, J. Dynamical and Control Systems, 14, 505-535. doi 10.1007/s10883-008-9049-5

[18] L. Mazzi and M. Sabatini, (1988), A characterization of centres via first integrals, J. Differential Equations, 76, 222237. doi 10.1016/0022-0396(88)90072-1

[19] R. Moussu, (1982), Symétrie et forme normale des centres et foyers dégénérés, Ergodic Theory and Dynamical Systems, 2, 241-251. doi 10.1017/S0143385700001553

[20] R. Moussu, (1982), Une démonstration d'un théorème de Lyapunov-Poincaré, Astérisque, 98-99, $216-223$.

[21] V.V. Nemytskii and V.V. Stepanov, (1989) Qualitative theory of differential equations, Dover Publ., New York.

[22] H. Poincaré, (1881), Mémoire sur les courbes définies par les équations différentielles, Journal de Mathématiques, 37, 375-422; Oeuvres de Henri Poincaré, vol. I, Gauthier-Villars, Paris, 1951, pp 3-84.

[23] E. Strózyna and H. Zoladek, (2002) The analytic and formal normal form for the nilpotent singularity, J. Differential Equations, 179, 479-537. doi 10.1006/jdeq.2001.4043

[24] F. Takens, (1947) Singularities of vector fields, Inst. Hautes Etudes Sci. Publ. Math., 43, 47-100. doi 10.1007/BF02684366

[25] M.A. Teixeira and J. Yang, (2001), The Center-focus Problem and Reversibility, J. Differential Equations, 174, 237251. doi 10.1006/jdeq.2000.3931

(C)The author. All rights reserved. 